\title{
AVALIAÇÃO DO CONFORTO TÉRMICO PARA OVINOS EM EXPOSIÇÃO DURANTE FEIRA AGROPECUÁRIA DE SANTARÉM
}

\author{
Jéssica de Carvalho Pantoja'; Cristiane Rebouças Barbosa²; Thaís Emanuely dos Santos Amaral³; Graciene \\ Conceição dos Santos ${ }^{4}$.

\footnotetext{
${ }^{1}$ Estudante de graduação Ciências Agrárias, Universidade Federal do Oeste do Pará (UFOPA), Instituto de Biodiversidade e Florestas (IBEF), Santarém, Pará, Brasil, jessicka.carvalho17@gmail.com

2 Estudante de graduação Ciências Agrárias, UFOPA, IBEF, Santarém, Pará, Brasil, cris_ag10@hotmail.com

3 Estudante de graduação Ciências Agrárias, UFOPA, IBEF, Santarém, Pará, Brasil, t.emanuely95@gmail.com

${ }^{4}$ Zootecnista e Professora Adjunta, UFOPA, IBEF, Santarém, Pará, Brasil, gracienecsantos@yahoo.com.br
}

RESUMO: A exposição de ovinos em feira agropecuária vem aumentando consideravelmente nos últimos anos, principalmente devido ao avanço de técnicas de manejo, nutrição e melhoramento genético. O presente trabalho objetivou-se avaliar a zona de conforto térmico em ovinos da raça Santa Inês e Dorper por meio de medições da temperatura, umidade relativa e respostas fisiológicas. O trabalho durou dois dias com a utilização de sete fêmeas de ambas as raças com idade média de sete meses. O experimento foi em delineamento experimental inteiramente casualizado em esquema fatorial 12x2. Para coleta das variáveis bioclimáticas utilizou-se a temperatura de bulbo seco, umidade relativa do ar, índice de temperatura e umidade, e as respostas fisiológicos foram frequência respiratória, frequência superficial (cabeça, tronco e garupa). Sob as condições ambientais adversas no local do experimento, os ovinos foram sujeitos ao estresse moderado, apresentando seus parâmetros fisiológicos acima da normalidade, em média, principalmente no período da tarde, estando fora da sua zona de termoneutralidade. A raça Santa Inês foi a que apresentou menor elevação da temperatura superficial, consequentemente, apontou maior tolerância ao estresse ambiental.

PALAVRAS-CHAVE: Ambiência, Bioclimatologia, Estresse.

\section{THERMAL COMFORT EVALUATON FOR SHEEP IN EXHIBITION DURING SANTARÉM AGRICULTURAL FAIR}

\begin{abstract}
The exhibition of sheep in agricultural fair has increased considerably in recent years, mainly due to the advancement of management techniques, nutrition and genetic improvement. The present study aimed to evaluate the thermal comfort zone in Santa Inês breed sheep and Dorper through measurements of temperature, relative humidity and physiological responses. The job lasted two days with seven females of both races with an average age of seven months. The experiment was in completely randomized experimental design in factorial scheme 12×2. Bioclimatic variables collection using the dry bulb temperature, relative humidity, temperature and humidity, and the physiological responses
\end{abstract}


were respiratory rate, often shallow (head, torso and back). Under adverse environmental conditions at the site of the experiment, the sheep were subjected to moderate stress, showing their physiological parameters above the normal range, on average, mainly in the afternoon, standing outside of your area of termoneutralidade. The Santa Inês breed was presented the lowest elevation of the surface temperature, consequently, pointed and increased tolerance to the environmental stress.

KEYWORDS: Bioclimatology, Environment, Stress.

\section{EVALUACIÓN DEL CONFORT TÉRMICO PARA OVINOS EN EXPOSICIÓN DURANTE FERIA AGROPECUARIA DE SANTARÉM}

RESUMEN: La exposición de ovejas en la Feria agrícola ha aumentado considerablemente en los últimos años, debido principalmente a la promoción de técnicas de manejo, nutrición y mejoramiento genético. El presente estudio pretende evaluar la zona de confort térmico en ovejas de raza Santa Inês y Dorper a través de mediciones de temperatura, humedad relativa y respuestas fisiológicas. El trabajo duró dos días con siete mujeres de ambas razas con una edad media de siete meses. El experimento fue en diseño experimental completamente al azar en esquema factorial $12 \times 2$. Colección de variables bioclimáticas utilizando la temperatura de bulbo seco, humedad relativa, temperatura y humedad y las respuestas fisiológicas eran la frecuencia respiratoria, a menudo poco profunda (cabeza, torso y espalda). Bajo condiciones ambientales adversas en el sitio del experimento, las ovejas fueron sometidas a estrés moderado, mostrando sus parámetros fisiológicos por encima del rango normal, en promedio, principalmente por la tarde, de pie fuera de su área de termoneutralidade. La raza Santa Inês se presentó la más baja elevación de la temperatura de la superficie, por lo tanto, acentuado y mayor tolerancia al estrés ambiental.

PALABRAS CLAVE: Bioclimatologia, Climación, Estrés.

A ovinocultura é uma atividade de grande importância social e econômica e vem apresentando um acentuado crescimento nos últimos anos no Brasil, seja como principal criação na propriedade rural, seja como secundária, em consórcio com outras produções de animais. Essa atividade expandiu também para o Norte do Brasil. Os animais introduzidos nesta região pertencem a diversas raças, destacando-se a raça Santa Inês e seus cruzamentos indiscriminados, animais SRD (Sem Raça Definida). 
No entanto, é necessário que o fator climático seja levado em consideração, pois as condições amazônicas apresentam-se como estressantes devido às altas temperaturas e também umidade relativa do ar durante todo o ano, em relação a outras regiões brasileiras. Isso destaca a necessidade do conhecimento da tolerância ao calor e capacidade de adaptação de cada raça à determinada região, servindo como embasamento técnico-científico para melhor desenvolver a atividade e também como critério de seleção para o crescimento vegetativo do rebanho (SILVA et al., 2010).

Apesar de ainda não haver muitos criadores de ovinos na cidade de Santarém, a criação ainda é fonte de renda para alguns produtores que investem nessa produção. A comercialização desses animais é realizada nas feiras agropecuárias da região, onde os produtores aproveitam para expor seus animais no período de realização do evento. Essa participação em feiras agropecuárias altera a rotina dos animais, que ficam potencialmente expostos a riscos. Os principais pontos críticos para o bem-estar dos animais que participam de exposições agropecuárias são os seguintes: tratamento dos animais nas propriedades antes da feira; embarque e transporte para o local do evento; desembarque dos animais no recinto da feira; alimentação e fornecimento de água durante todo o período da feira (qualidade da dieta e mudança da rotina); exposição dos animais aos ambientes sonoro, visual e olfativo atípicos das condições de criação e consequentemente estressante; estresse durante o julgamento e embarque e transporte de volta para a propriedade (ZANELLA, 2001).

Em feiras agropecuárias, é existente a preocupação por parte dos criadores a respeito do local e forma como os animais ficam acomodados durante o período em que aguardam para serem julgados e comercializados, uma vez que, a proximidade com o público, ruído, o tipo de instalações, acesso à água e alimento, podem comprometer o seu comportamento e conseguinte, o seu desempenho (DINIZ et al., 2014).

Dentre os fatores que influenciam no 
desempenho da produção animal, temse o clima que afeta direta e indiretamente os animais. Como os ovinos são animais homeotérmicos, possuindo a capacidade de controlar sua temperatura corporal, em uma temperatura ambiente dentro de certos limites, é essencial a provisão de instalações que mantenham as temperaturas ambientais próximas as das condições de conforto térmico para os animais (ROBERTO et al., 2014).

Várias são as formas de verificar se os animais se encontram em condições de estresse, dentre eles tem-se as alterações fisiológicas e as comportamentais. No que se refere às fisiológicas, cita-se a temperatura corporal como um fator que pode levar a um maior consumo de energia, desviado na maioria das vezes, das suas funções reprodutiva e produtivas (DINIZ et al., 2014). Os animais ainda em condição de estresse e desconforto podem aumentar a frequência respiratória, diminuir o consumo de alimento e aumentar o consumo de água.

Mensurações de variáveis fisiológicas, índices de conforto térmico e observações comportamentais são ferramentas muito utilizadas em pesquisas nas áreas de ambiência. E no contexto amazônico, a umidade relativa do ar assume um importante papel na determinação do conforto térmico dos animais. Pois, em uma mesma temperatura ambiente, com umidade relativa diferente, as sensações térmicas são alteradas, podendo o animal estar em estresse térmico, ou não (FERREIRA, 2004).

Tendo em vista que o clima pode interagir com os animais alterando sua resposta fisiológica, comportamental e produtiva, objetivou-se avaliar o conforto térmico dos ovinos expostos no ambiente da Feira Agropecuária de Santarém, Pará.

O trabalho foi desenvolvido no Parque de Exposições Alacid Nunes, localizado na Avenida Antônio Simões, bairro da Prainha no município de Santarém - Pará, latitude 020 25' 56" S e longitude 540 41' 27" W, com média de temperatura anual entre de $25^{\circ} \mathrm{C}$ a $28^{\circ} \mathrm{C}$, umidade relativa média do ar de 86\%, tendo como clima dominante na região o quente e úmido, típico que regiões tropicais (IBGE, 2015).

Foram utilizados 14 ovinos, sete da raça 
Dorper com média de peso de $55 \mathrm{~kg}$ e sete da raça Santa Inês com peso médio de 51 kg, fêmeas com idade média de 7 meses, distribuídos homogeneamente em duas baias.

Durante o experimento, os animais receberam água à vontade, silagem de milho e concentrado com farelo de trigo comercial, de acordo com a categoria e o estádio fisiológico dos animais, com ofertas de duas vezes ao dia. As variáveis ambientais e os parâmetros fisiológicos dos ovinos foram aferidos a cada duas horas em um período de 48 horas, no mês de agosto do ano de 2017.

As respostas fisiológicas dos animais foram coletadas através da temperatura superficial e frequência respiratória (mov. Min - 1). Os dados foram coletados sem interferência sobre os animais.

A frequência respiratória (FR) foi aferida através da contagem dos movimentos respiratórios no flanco dos animais, por meio de um cronômetro, durante 20 segundos, e os valores coletados multiplicados por três para cálculo da FR minuto-1.

Para temperatura superficial foram coletados em três pontos (cabeça, tronco e garupa) com o auxílio de um termômetro de infravermelho, sendo posicionado aproximadamente a um metro de distância do animal; e as variáveis ambientais registraram-se por meio de um termo higrômetro digital da marca Instrutemp ${ }^{\circledR}$, modelo ITHT 2250 para determinar a caracterização do microclima.

Conforme executado por Silva (2010), foram coletadas as variáveis ambientais. $\bigcirc$ cálculo do Índice de temperatura e umidade (ITU) foi obtido, de acordo com Buffington et al. (1981), a partir da fórmula: ITU = Tbs + 0,36Tpo + 41,2.

O experimento foi conduzido em delineamento experimental inteiramente casualizado (DIC) em esquema fatorial 12 x 2 (horários de coleta e dois grupos de animais) em 48 horas de exposição para cada tratamento. As comparações entre as médias foram realizadas por meio do teste de Tukey, com níveis de probabilidade de $5 \%$. Os resultados foram obtidos por meio do programa Sisvar 5.3.

Os valores médios de temperatura ambiente (tbs), umidade relativa do ar 
(UR) e o índice de temperatura e umidade experimental, estão apresentados na (ITU), obtidos durante o período Tabela 1.

Figura 1. Representação das medidas de temperatura superficial.

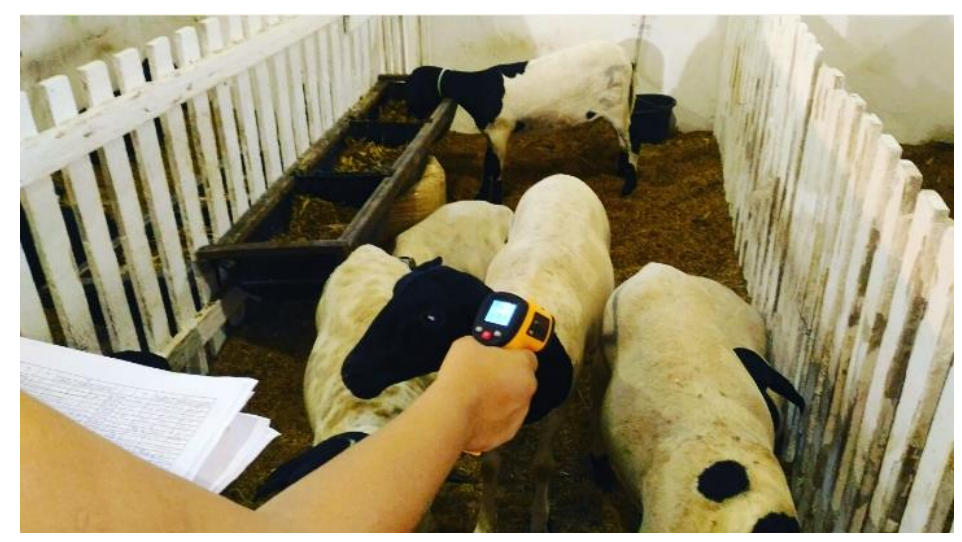

Fonte: Autor próprio

Tabela 1. Médias da temperatura ambiente (tbs), Umidade Relativa do Ar - UR \% e do Índice de Temperatura e Umidade (ITU), em ambiente de exposição de ovinos, durante feira agropecuária.

\begin{tabular}{cccc}
\hline Horários & Tbs $\left({ }^{\circ} \mathrm{C}\right)$ & UR $(\%)$ & ITU \\
\hline 1 & $28,16 \mathrm{~h}$ & $72,00 \mathrm{a}$ & $77,50 \mathrm{c}$ \\
3 & $28,00 \mathrm{i}$ & $72,00 \mathrm{a}$ & $77,29 \mathrm{~b}$ \\
5 & $27,90 \mathrm{j}$ & $71,50 \mathrm{~b}$ & $77,11 \mathrm{a}$ \\
7 & $28,55 \mathrm{~g}$ & $67,50 \mathrm{c}$ & $77,64 \mathrm{c}$ \\
9 & $29,90 \mathrm{e}$ & $63,50 \mathrm{f}$ & $79,08 \mathrm{e}$ \\
11 & $32,40 \mathrm{c}$ & $54,50 \mathrm{~h}$ & $81,53 \mathrm{~h}$ \\
13 & $33,60 \mathrm{~b}$ & $54,00 \mathrm{i}$ & $83,08 \mathrm{k}$ \\
15 & $34,55 \mathrm{a}$ & $48,50 \mathrm{j}$ & $83,70 \mathrm{I}$ \\
17 & $33,60 \mathrm{~b}$ & $47,50 \mathrm{k}$ & $82,32 \mathrm{j}$ \\
19 & $31,45 \mathrm{~d}$ & $55,50 \mathrm{~g}$ & $80,37 \mathrm{~g}$ \\
21 & $29,90 \mathrm{e}$ & $64,00 \mathrm{e}$ & $79,14 \mathrm{f}$ \\
23 & $29,90 \mathrm{e}$ & $66,00 \mathrm{~d}$ & $78,37 \mathrm{~d}$ \\
\hline Média Geral & 30,60 & 61,37 & 79,76 \\
\hline
\end{tabular}

As médias com mesma letra nas colunas não diferem significativamente entre si pelo teste de Tukey $(P<$ 0,05). Fonte: Autor próprio.

Verificou-se que os maiores valores identificados do Tbs foram no período da tarde, nos horários de 15, 13 e 11

horas respectivamente, resultado esse 
atribuído provavelmente a maior incidência de radiação solar à tarde e confirmado por Andrade et al. (2007).

Observou-se que as médias da temperatura ambiente ficaram acima e dentro da zona de conforto térmico (ZCT) para a espécie, que segundo Baêta e Souza (2010) deve situar-se entre 20 e $30^{\circ} \mathrm{C}$. Considerando estes valores, conclui-se que os horários de $11,13,15,17$ e 19 horas, se encontraram acima da ZCT, o que pode afetar os índices fisiológicos e produtivos dos animais, assim também como no comportamento.

Júnior et al. (2016) em trabalho com ovinos na Paraíba, citam temperaturas elevadas, o que afetou os índices fisiológicos e produtivos dos animais. Avaliando a umidade relativa constatou-se que houve uma variação entre 47,50 \% e 72\% nos horários 17, 1 e 3 horas respectivamente. Como resultado a média geral da UR teve o valor de $61,37 \%$ estando dentro das condições ideais, segundo estudo realizado por Júnior et al. (2016), que considerou a UR ideal para ovinos entre 60 e 70\%. Nos horários 11, 13, 15, 17,19 se encontram abaixo da UR ideal para os animais. Vale ressaltar que a média de UR da região de Santarém é de 86\% segundo Silva et al. (2016).

As maiores médias nos valores de ITU foram encontradas nos horários de 15 e 13 horas da tarde, com valores de 83,70 e 83,08, podendo ser melhor observado na evolução horária do ITU na Figura 2. Esse resultado indica que os animais sofreram um estresse moderado nesses horários, conforme escala apresentada por Neves (2008), onde menor que 82 aponta ausência do estresse de calor, de 82 a menor que 84 indica estresse moderado, de 84 a menor que 86 o estresse é considerado severo e a partir de 86 estresse de calor extremamente severo.

O resultado deste trabalho assemelha-se com o encontrado por Neves et al. (2009) com o valor máximo de ITU de 82,2 no período da tarde considerado como estresse moderado, em estudo com ovinos da raça Santa 
Inês. Porém, os valores aqui relatados são, em média, inferiores ao relatado em outro estudo no clima amazônico, onde o valor médio foi de 84,85, indicando que os animais sofreram um estresse severo ao calor (COSTA et al., 2010).

Figura 2. Índice de Temperatura e Umidade (ITU) nos diferentes horários do dia.

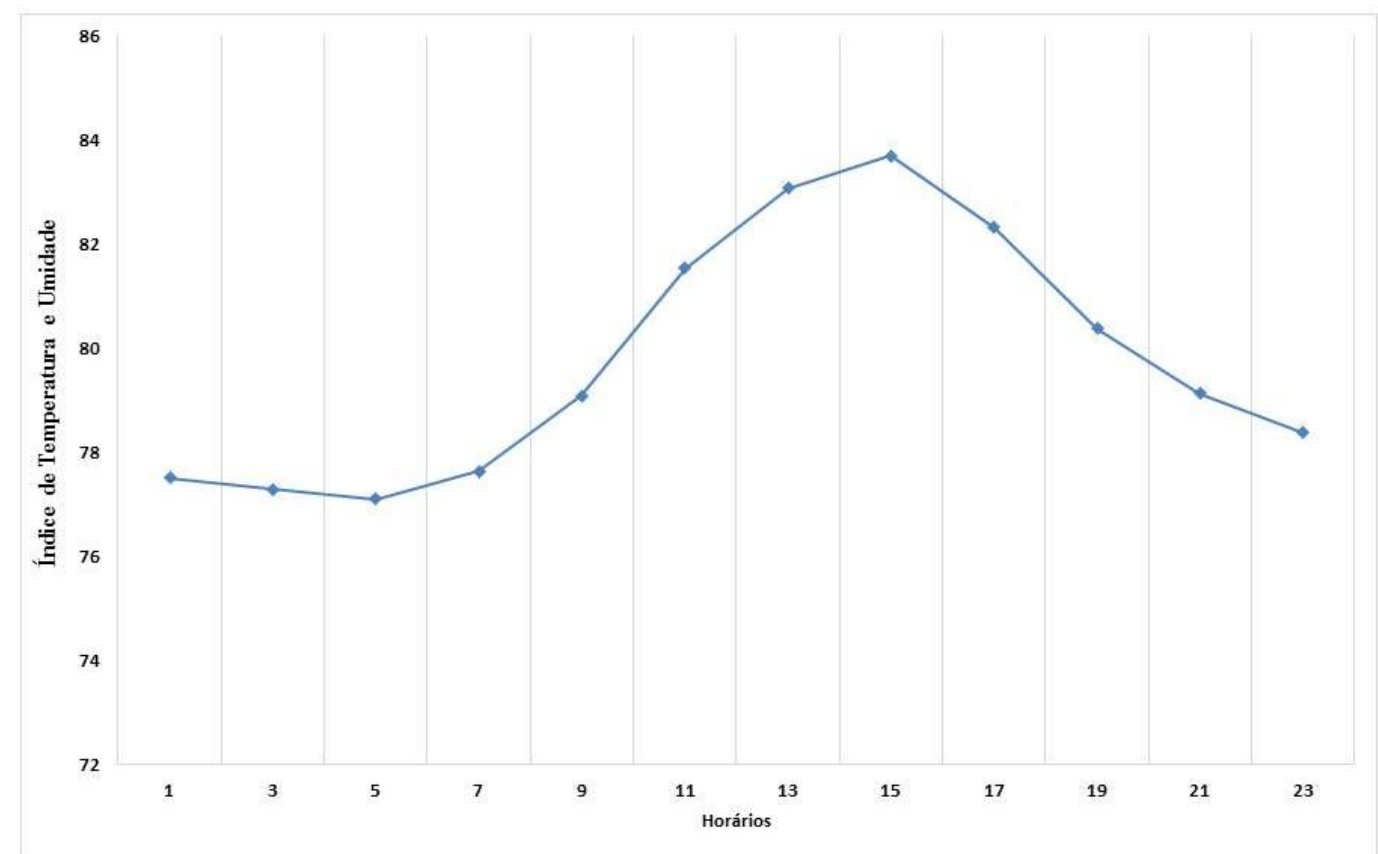

Fonte: Autor próprio

A TS e a FR são parâmetros utilizados para medir o estresse térmico em animais e funcionam como indicadores homeostáticos para dissipação de calor, por evaporação respiratória e cutânea. Cezar et al. (2004) indica que a sudorese é menos importante do que a evaporação respiratória para os ovinos e quando esses são expostos a elevadas temperaturas, a taxa respiratória aumenta. Na Tabela 2 são apresentados os valores médios, da frequência respiratória (FR) e temperatura superficial (TS) das raças Dorper e Santa Inês em todos os horários avaliados. 
Tabela 2. Médias da frequência respiratória (FR) e temperatura superficial (TS) das raças Dorper e Santa Inês, em exposição feira agropecuária.

\begin{tabular}{|c|c|c|c|c|c|c|}
\hline & FR & & & TS & & \\
\hline Horários & Dopper & Santa Inês & Média & Dopper & Santa Inês & Média \\
\hline 1 & $76,50 b$ & $96,00 a$ & $86,25 \mathrm{~g}$ & $33,56 a$ & $30,20 b$ & $31,88 \mathrm{k}$ \\
\hline 3 & $81,00 b$ & $84,21 a$ & $82,60 \mathrm{~h}$ & $35,94 a$ & $29,16 b$ & $32,55 j$ \\
\hline 5 & $65,36 b$ & $91,71 a$ & $78,53 j$ & $35,15 a$ & $30,35 b$ & $32,75 i$ \\
\hline 7 & $62,14 b$ & $67,50 a$ & 64,821 & $33,28 a$ & $30,13 b$ & 31,701 \\
\hline 9 & $71,57 b$ & $79,29 a$ & $75,43 \mathrm{k}$ & $37,52 a$ & $31,69 b$ & $34,60 \mathrm{~g}$ \\
\hline 11 & $94,93 b$ & $104,12 a$ & $99,53 c$ & $39,49 a$ & $34,07 b$ & $36,78 d$ \\
\hline 13 & $88,71 b$ & $105,21 a$ & $96,96 \mathrm{e}$ & $40,23 a$ & $35,73 b$ & $38,61 \mathrm{c}$ \\
\hline 15 & $106,71 a$ & $103,93 b$ & $105,32 b$ & $41,16 a$ & $36,06 b$ & $40,85 b$ \\
\hline 17 & $80,79 b$ & $82,07 a$ & $81,43 i$ & $43,71 a$ & $37,99 b$ & $40,85 a$ \\
\hline 19 & $77,36 b$ & $102,00 a$ & $89,68 f$ & $38,67 a$ & $34,06 b$ & $36,36 \mathrm{e}$ \\
\hline 21 & $100,50 b$ & $112,93 a$ & $106,71 a$ & $37,00 a$ & $32,78 b$ & $34,89 f$ \\
\hline 23 & $88,07 b$ & $110,57 a$ & $99,32 d$ & $36,50 a$ & $31,40 b$ & $33,95 h$ \\
\hline Média Geral & $82,89 b$ & $94,96 a$ & & $37,68 a$ & $32,80 b$ & \\
\hline
\end{tabular}

Na média geral a FR foi diferente estatisticamente em todos os horários observados, a maior FR foi às 21 horas com valor de 106,71 mov. min-1 e a menor foi às 7 horas com valor de 64,82 mov. min-1. A raça Santa Inês obteve taxas maiores de FR em relação à Dorper, exceto no horário de 15 horas, onde a raça Dorper teve valor 106,71 mov. min-1, superior ao da Santa Inês com valor de 103,93 mov. min-1. Este resultado difere do encontrado por Cezar et al. (2004), onde a raça Dorper obteve FR superior em relação à Santa Inês.

Ambas as raças sofreram estresse alto de acordo com a escala utilizada por Filho (2011), onde 40 a 60 - estresse baixo, 60 a 80 - estresse médio-alto e 80 a 120 mov. min-1 considerado estresse alto. $\bigcirc$ valor considerado mais estressante foi no horário de 21 horas, onde a taxa de respiração foi a maior para as duas raças, com valores de 
112,93 mov. min-1 para Santa Inês e 100,50 para Dorper. O horário de 7 horas apresentou dados menores de frequência respiratória para as respectivas raças, no entanto estes valores de 62,14 e 67,50 apresentam ainda estresse médio-alto para esses animais, indicando que em todos os tratamentos avaliados os ovinos não estavam em conforto térmico. Os dados encontrados neste trabalho são superiores que os citados por Neves (2009), Andrade et al. (2007), Júnior et al. (2016) e Oliveira (2013) com valores de 60,$0 ; 61,64,55,0$ e 54,98 mov. min-1 respectivamente.

A raça Dorper apresentou a maior temperatura superficial em todos os tratamentos com ponto máximo de 43,71 ${ }^{\circ} \mathrm{C}$ no horário de 17 horas, diferindo da raça Santa Inês $(P<0,05)$. Quando se desdobrou a interação da raça dentro dos horários mostrou diferença $(P<0,05)$ para ambas as raças. Em estudos realizado, por Batista et al. (2014) verificou-se resultados distintos dos encontrados neste trabalho, onde a raça Santa Inês teve uma maior temperatura superficial comparada a raça Dorper. Observouse que as maiores médias de temperaturas foram pela parte da tarde, nos horários de 1715 e 13 horas respectivamente para as duas raças. Resultado ocasionado provavelmente devido as maiores temperaturas encontradas nos mesmos horários. Esses resultados corroboram com o estudo realizado por Pires et al. (2016) que também observaram as maiores médias de temperatura superficial no turno da tarde.

Esta elevação da TS é uma maneira dos ovinos dissiparem calor para o meio ambiente através da vasodilatação periférica, em que o gradiente entre o núcleo corporal e a pele se eleva enquanto o gradiente térmico entre a pele e o ambiente diminui (FILHO et al 2014).

Na Figura 3 pode-se observar a influência da UR, do Tbs na temperatura superficial de ambas as raças. 
Figura 3. Influência da Umidade Relativa (UR) e Temperatura do bulbo seco (Tbs) na temperatura superficial (TS) das raças Dorper e Santa Inês.

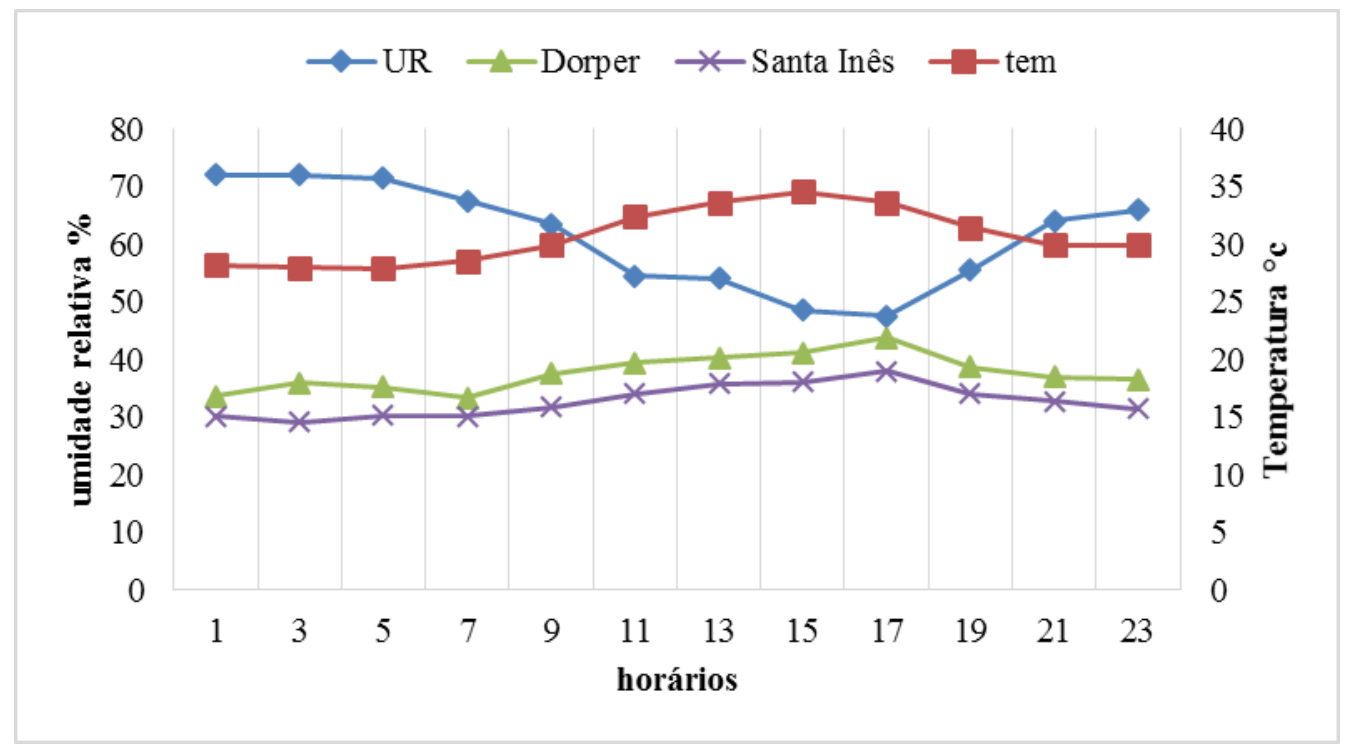

Fonte: Autor próprio.

A umidade relativa varia em função da temperatura do ar, diminuindo com o aumento desta. Quando o ar, contendo certa quantidade de água é esfriado, sua capacidade de reter água é reduzida, aumentando a UR até se tornar saturado (RIBEIRO, 2006). Os valores de Tbs e UR foram inversamente proporcionais, portanto, quando a temperatura ambiente registrou maior média, a umidade relativa expressou a média mínima encontrada. Tais resultados indicaram a elevação da temperatura superficial nas duas raças no horário de 17 horas, demonstrando uma possível tentativa de dissipação de calor através da evaporação para o ambiente.

Silva (2013), afirma que a manutenção da temperatura corporal ocorre mediante trocas de calor com o ambiente, sendo que, em temperaturas mais amenas, o calor é dissipado para o ambiente na forma sensível, através de um gradiente de temperatura entre o animal e o meio ambiente, e sob estresse pelo calor, o principal processo de perda é o da evaporação.

As menores médias de temperatura superficial nas raças foram quando a 
temperatura ambiente estava menor e a umidade relativa elevada, nos horários entre 1 e 9 horas da manhã, Sob as condições ambientais adversas, encontradas no local da feira agropecuária de Santarém, os ovinos tiveram estresse moderado, apresentando, parâmetros fisiológicos acima da normalidade, principalmente

\section{REFERÊNCIAS}

ANDRADE, I. S; SOUZA, B. B; FILHO, J. M. P; SILVA, A. M. A. Parâmetros fisiológicos e desempenho de ovinos Santa Inês submetidos a diferentes tipos de sombreamento e a suplementação em pastejo. Ciência e Agrotecnologia, Lavras, v. 31, n. 2, p. 540-547, mar./abr., 2007.

BAÊTA, F. C:; SOUZA, C. F. Ambiência em edificações rurais: Conforto animal. 2.ed. Viçosa: UFV, 2010. 269p

BATISTA, N. L; SOUZA, B. B; OLIVEIRA, G. J. C; ROBERTO, J. V. B; ARAUJO, R. P; RIBEIRO, T. L. A; SILVA, R. A. Tolerância ao calor em ovinos de pelames claro e escuro submetidos ao estresse térmico.

J Anim Behav Biometeorol, v. 2, n. 3, p. 102- 108, 2014.

BUFFINGTON, D. E.; COLLAZOAROCHO, A.; CANTON, G.H.; PITT, D.; THATCHER, W.W.; COLLIER, R.J. Black globe-humidity index (BGHI) as sendo este período, a melhor faixa de conforto para as raças analisadas, neste experimento.

no período da tarde, estando fora da sua zona de termoneutralidade. De acordo, com as variáveis mensuradas a raça Santa Inês foi a que expressou maior tolerância as condições ambientais da exposição.

comfort equation for dairy cows. Transactions of ASAE, v. 24, n. 3, p.711714, 1981.

CEZAR, M. F; SOUZA, B. B; SOUZA, W. H; FILHO, E. C. P; TAVARES, G. P; MEDEIROS, G. X. Avaliação de parâmetros fisiológicos de ovinos Dorper, Santa Inês e seus mestiços perante condições climáticas do trópico semi-árido nordestino. Ciência e Agrotecnologia, Lavras, v. 28, n. 3, p. 614-620, maio/jun., 2004.

COSTA, E. P. S; CRUZ, T. F. R. P.; SANTOS, L. R. Avaliação da Adaptabilidade de Ovinos Santa Inês ao Clima Amazônico. REDVET. Revista electrónica de Veterinaria, v. 11, n. 3, p. 1695, 2010.

DINIZ, T. A; SILVA, G. C; CARVALHO, C. C. S; PEREIRA, K. C. B; CASTRO, A. L. O; BRITO, S. N; JUNIOR, I. O. A. Temperatura corporal de Equinos 
Alojados em Diferentes Baias, 2014. In: FÓRUM ENSINO, PESQUISA, EXTENSÃO E GESTÃO, 8. Brasília. Anais... Brasília: FEPEG, 2014.

FERREIRA, R.A.; FILHO, E. C. P.; TAVARES, G. P.; MEDEIROS, G. X. Avaliação de parâmetros fisiológicos de ovinos Dorper, Santa Inês e seus mestiços perante condições climáticas do trópico semi-árido nordestino. Ciência e Agrotecnologia, Lavras, v. 28, n. 3, p. 614-620, maio/jun., 2004.

FILHO, J. R. M.; FURTADO, D. A.; NASCIMENTO, J. W. B.; OLIVEIRA, C. J. B. Produção, qualidade do leite e índices fisiológicos de cabras Alpinas no semiárido no período chuvoso. Revista Brasileira de Engenharia Agrícola e Ambiental v.18, n.7, p.762768, 2014.

IBGE - Instituto Brasileiro de Geografia Estatística. Disponível em: $<$ http://mapas.ibge.gov.br/bases-ereferenciais/bases-

cartograficas/malhas-digitais>. Acesso em: 04 Set. 2017.

JÚNIOR, J. J. L. M; SILVA, D. A. F. W. S; LEITE, P. G; NETO, S. G. Índices de conforto térmico e respostas fisiológicas de ovinos mestiços confinados recebendo água salina, 2016. In: Congresso Técnico Científico da Engenharia e da Agronomia, 73. Foz do Iguaçu. Anais... Foz do Iguaçu: CTCEA, 2016.

NEVES, M. L. M. W. Índices de conforto térmico para ovinos Santa Inês de diferentes cores de pelame em condições de pastejo. 2008. $77 \mathrm{f}$. Dissertação (Programa de PósGraduação em Zootecnia) Universidade Federal Rural de Pernambuco, Recife.

NEVES, M. L. M. W; AZEVEDO, M; BORGES, L. A. B; GUIM, A; LEITE, A. M; CHAGAS, J. C. Níveis críticos do Índice de Conforto Térmico para ovinos da raça Santa Inês criados a pasto no agreste do Estado de Pernambuco. Acta Scientiarum. Animal Sciences Maringá, v. 31, n. 2, p. 169-175, 2009.

OLIVEIRA, F. A; TURCO, S. H. N; BORGES, I.; CLEMENTE, C. A. A; NASCIMENTO, T. V. C; FILHO, J. B. L. Parâmetros fisiológicos de ovinos Santa Inês submetidos a sombreamento com tela de polipropileno. Revista Brasileira de Engenharia Agrícola e Ambiental, v. 17, n. 9, p. 1014-1019, 2013.

PIRES, J. P. S; DANTAS, N. L. B; SILVA, M. R.; BATISTA, L. QUEIROZ, E. O.; MACEDO, F. A. F; BARBOSA, O. R.; ZANCANELA, V; MORA, N. H. A. P.; BALISCEI, M. A. Parâmetros fisiológicos e desempenho para ovelhas Santa Inês e cordeiros 1/2 Dorper - Santa Inês nas estações verão e inverno. Revista Brasileira de Saúde e Produção Animal, Salvador, v. 16, n. 1, p.199-209 jan./mar., 2015.

RIBEIRO, N. L. Avaliação do conforto térmico de Ovinos nativos em confinamento, Campina Grande, 2006. Dissertação (Mestrado em Construções Rurais e Ambiência)- Universidade 
Federal de Campina Grande, Curso de Pós-Graduação em Engenharia Agrícola, do Centro de Tecnologia e Recursos Naturais, 2006.

ROBERTO, J. V. B; SOUSA, B. B; NOBRE, I. S; LEUCENA, J. E. S; LIMA, R. D. Variação dos elementos climáticos e respostas termorreguladoras de caprinos no semiárido brasileiro. J Anim Behav Biometeorol v. 2, n. 4, p.131-138, 2014.

SILVA, G. A. Avaliação do sistema de resfriamento adiabático evaporativo na melhoria do bem-estar de novilhas leiteiras em confinamento, 2016. Dissertação (Mestrado em Produção Animal Sustentável)- Programa de Pósgraduação do Instituto de Zootecnia, 2013.

SILVA, M. A. G; JUNIOR, J. M. G; SILVA, N. F. C; SANTOS, F. C. V; UCKER, F. E. Caracterização pluviométrica de Santarém-PA, Brasil. Revista eletrônica de educação da faculdade araguaia, $v$. 10, p. 112-120, 2016.

ZANELLA, A. J. Considerações éticas relacionadas com a utilização de animais em feiras agropecuárias. A Hora Veterinária, n. 123, p. 58, Set. 2001. 